\title{
Numerical study of a magnetically insulated front-end channel for a neutrino factory
}

\author{
Diktys Stratakis, ${ }^{*}$ Richard C. Fernow, Juan C. Gallardo, and Robert B. Palmer \\ Department of Physics, Brookhaven National Laboratory, Upton, New York 11973, USA \\ David V. Neuffer \\ Fermi National Accelerator Laboratory, Batavia, Illinois 60510, USA
}

(Received 24 August 2010; published 14 January 2011)

\begin{abstract}
A neutrino factory, which can deliver an intense flux of $\sim 10^{21}$ neutrinos per year from a multi-GeV stored muon beam, is seemingly the ideal tool for studying neutrino oscillations and $C P$ violations for leptons. The front end of this facility plays a critical role in determining the number of muons that can be accepted by the downstream accelerators. Delivering peak performance requires transporting the muon beams through long sections of a beam channel containing high-gradient $\mathrm{rf}$ cavities and strong focusing solenoids. Here, we propose a novel scheme to improve the performance of the cavities, thereby increasing the number of muons within the acceptance of the accelerator chain. The key element of our new scheme is to apply a tangential magnetic field to the rf surfaces, thus forcing any field-emitted electrons to return to the surface before gaining enough energy to damage the cavity. We incorporate this idea into a new lattice design for a neutrino factory, and detail its performance numerically. Although our proposed front-end channel requires more rf power than conventional pillbox designs, it provides enough beam cooling and muon production to be a feasible option for a neutrino factory.
\end{abstract}

DOI: 10.1103/PhysRevSTAB.14.011001

PACS numbers: 29.27.-a, 29.20.Ej, 41.75.Lx

\section{INTRODUCTION}

Recent experiments demonstrated that neutrinos can oscillate among their different flavors [1], indicating that they are massive bodies. Such results have important consequences for the standard model, the theory that describes fundamental particles and their interactions, wherein neutrinos were assumed to be massless particles. However, most observations so far have been of naturally occurring neutrinos striking the Earth from the Sun and from supernova. It was suggested [2] that neutrinos from the decay of a stored high-energy muon beam (in a neutrino factory) are the ultimate tool for studying neutrinos and their oscillations in far greater detail since they would constitute a much more intense and precisely defined beam for researchers.

In a neutrino factory [3-12], 5-15 GeV bunches of protons are focused onto a target [13] to produce pions that decay into muons, which are then accelerated to energies up to a few tens of GeV. The muons are subsequently stored in a racetrack-shaped storage ring, where their decays provide intense beams of neutrinos. A key requirement in maximizing the final flux of neutrinos is that the phase-space volume of the muon beams must match the acceptance of the downstream accelerators. This demands a front-end channel (the part of the facility between the target and the first linear accelerator) for manipulating the beam in transverse and longitudinal phase space. For the latter, a series of $\mathrm{rf}$ cavities form the resulting muon

\footnotetext{
*Present address: Department of Physics \& Astronomy, University of California, Los Angeles, CA 90095.
}

beam into strings of bunches with different energies, and then align them into nearly equal central energies by phase rotating the beam. To reduce the volume of transverse phase space, the bunches are passed through a cooling channel, which reduces the beam's emittance using ionization cooling [14]. The cooling channel consists of absorbers, which lower the transverse and longitudinal momentum of beam particles, and rf cavities, which only restore the particle's longitudinal momentum, inside a magnetic channel. Since the cost of the front end is roughly one-third of the total cost of the factory complex, it is critically important to assure that its performance maximizes efficiency while reducing its risk.

It is important to emphasize that the front-end lattices designed so far require the rf cavities to operate within strong magnetic fields that focus the muons. For instance, typical rf gradients in the cooling channel are $15 \mathrm{MV} / \mathrm{m}$, while the magnetic field alternates between -2.8 to $2.8 \mathrm{~T}$. Experimental studies indicated that this configuration enhances the possibility of $\mathrm{rf}$ breakdown [15]. Hence, it is likely that the rf gradient will be limited by the magnetic field, and it is uncertain whether we can achieve the gradients required for the phase rotation and cooling sections. For this reason it is important to examine possible mitigation techniques, so that we can avoid possible rf gradient limitations due to the presence of strong magnetic fields.

It has been suggested experimentally $[16,17]$ and numerically $[18,19]$ that the causes of the rf operational problems in strong magnetic fields are field-emitted electrons that are accelerated to the order of an $\mathrm{MeV}$ and 
focused onto other surfaces. One promising solution to this problem is to apply magnetic insulation to the system. Magnetic insulation is an established technique for inhibiting breakdown in dc or pulsed voltage applications [20]. Recently, extension of the concept to low frequency rf cavities (201-805 MHz) has been suggested. Computations have shown that, with this configuration, damage mechanisms which result from field-emission processes can be effectively suppressed since the magnetic field forces the emitted electrons to return to the surface before gaining enough energy to damage the cavity [21].

The idea of magnetic insulation has been recently tested in an $805 \mathrm{MHz}$ box cavity $[16,17]$. The cavity was placed within a $3 \mathrm{~T}$ solenoid and mounted on an adjustable support so that the angle between the rf electric field and the external magnetic field could be varied. Compared to the case where there was a component of a $0.2 \mathrm{~T}$ field parallel to the rf electric field, the first preliminary results showed a $30 \%$ larger maximum gradient threshold when the magnetic field was introduced at right angles to the rf electric field. In this paper we present results of our simulations extending the concept of magnetic insulation to the frontend lattice of a neutrino factory, and compare its performance against the baseline lattice with conventional pillbox cavities. We propose a front-end lattice configuration that employs rf accelerating cavities with focus coils on either side. The shape of the cavity walls closely follows the magnetic-field lines on all surfaces with significant $\mathrm{rf}$ surface gradients. We show that in this novel structure all field-emitted electrons will be returned by the magnetic field to their surface of origin with only small nondamaging energies of less than $0.5 \mathrm{keV}$.

Our findings demonstrate that our proposed channel offers a satisfactory alternative option for the front-end of a neutrino factory. However, this magnetically insulated channel likely has higher rf power consumption than a similar channel using pillbox rf cavities. We also indicate the sensitivity of the channel performance to variations in the design parameters. For instance, we show that the lattice performance is not strongly affected by a threefold reduction in the number of discrete rf frequencies. Finally, we suggest key optimizations to reduce the heating on the absorber windows.

The layout of this paper is as follows: In Sec. II, we give an overview of the conventional baseline of the neutrino factory front end. In Sec. III we discuss the concept of magnetic insulation, and in Sec. IV we demonstrate an alternative option for a front end based on magnetically insulated cavities. Finally, we present our conclusions in Sec. V.

\section{FRONT-END BASELINE FOR A NEUTRINO FACTORY}

In the remainder of this paper we describe the neutrino factory front end and discuss its expected performance.
Detailed studies on a neutrino factory front-end design have been ongoing for the past few years $[5,22,23]$. Recently, the International Design Study for a Neutrino Factory (IDS-NF) proposed a baseline machine configuration [24]. Using this baseline, the parameters of the neutrino factory are being developed to deliver $\sim 10^{21}$ muon decays per operational year of $10^{7}$ secs with which to precisely measure neutrino oscillations. Based on the IDS-NF recommendations, the channel will lower the rms transverse emittance, $\varepsilon_{t}$ by a factor of $\sim 3$. The accepted muons per proton, $\mu / p$, will rise by a factor of $\sim 7-8$ within a transverse normalized acceptance, $A_{\mathrm{T}}$ less than $0.03 \mathrm{~m}$ and a longitudinal acceptance, $A_{L}$ less than $0.15 \mathrm{~m}$. The chosen acceptance criteria approximate the expected acceptance of the downstream acceleration and storage rings.

Figure 1 schematically represents the IDS-NF front-end scheme. In this configuration, a $4 \mathrm{MW}$ proton driver produces bunches with length in the range of 1-3 ns and $8 \mathrm{GeV}$ in energy. The beam is directed onto a $\mathrm{Hg}$ jet target enclosed in a $20 \mathrm{~T}$ solenoid. The pions created are captured as they transverse a $\sim 15 \mathrm{~m}$ long, tapered superconducting solenoidal magnet system, where the field profile drops adiabatically from 20 to $1.5 \mathrm{~T}$. Simultaneously, the radius of the beam pipe increases from $7.5 \mathrm{~cm}$ at the target up to $\sim 30 \mathrm{~cm}$ at the end of the taper. This is followed by a $70 \mathrm{~m}$ drift section with a constant $1.5 \mathrm{~T}$ field, where the pions decay into muons, and the beam develops a time-energy correlation with a high-energy "head" and a low-energy "tail."

The decay channel is followed by a $33 \mathrm{~m}$ buncher section [Fig. 2(a)] in which the gradient of the rf system

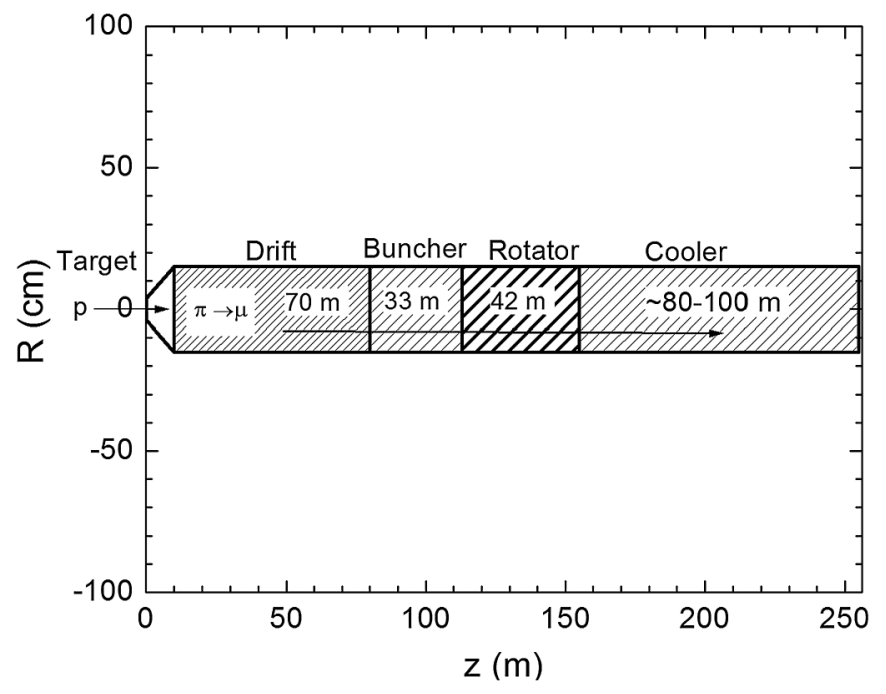

FIG. 1. Schematic representation of the front-end system of a neutrino factory, showing a target, a drift, an rf buncher, a phaseenergy rotator, and a cooler section. Pions are produced by protons on a target at the beginning of the drift, decay to muons in the drift, and while lengthening in phase, the buncher and rotator change the muons into a string of bunches with nearly equal energies. 
gradually increases and the beam is captured into a string of bunches with different energies. The rf frequency decreases along the length of the buncher, with the constraint that the phase difference between two reference particle momenta, $p_{1}$ and $p_{2}$, remains a fixed number $N_{B}$ of wavelengths as the beam propagates through it, i.e.,

$$
N_{B} \lambda_{r f}(z)=z\left(\frac{1}{\beta_{2}}-\frac{1}{\beta_{1}}\right)
$$

where $\beta_{1}$ and $\beta_{2}$ are the velocities of the reference particles at momentum $p_{1}$ and $p_{2}$. Following this procedure, the reference particles and all intermediate bunch centers remain at zero crossings of the rf wave throughout the buncher. For the present IDS-NF baseline design [24], $p_{1}=233 \mathrm{MeV} / \mathrm{c}$ and $p_{2}=154 \mathrm{MeV} / \mathrm{c}$ and $N_{B}=10$. With these parameters, the $\mathrm{rf}$ frequency at the beginning of the buncher section is $325.6 \mathrm{MHz}$ and at the end falls to
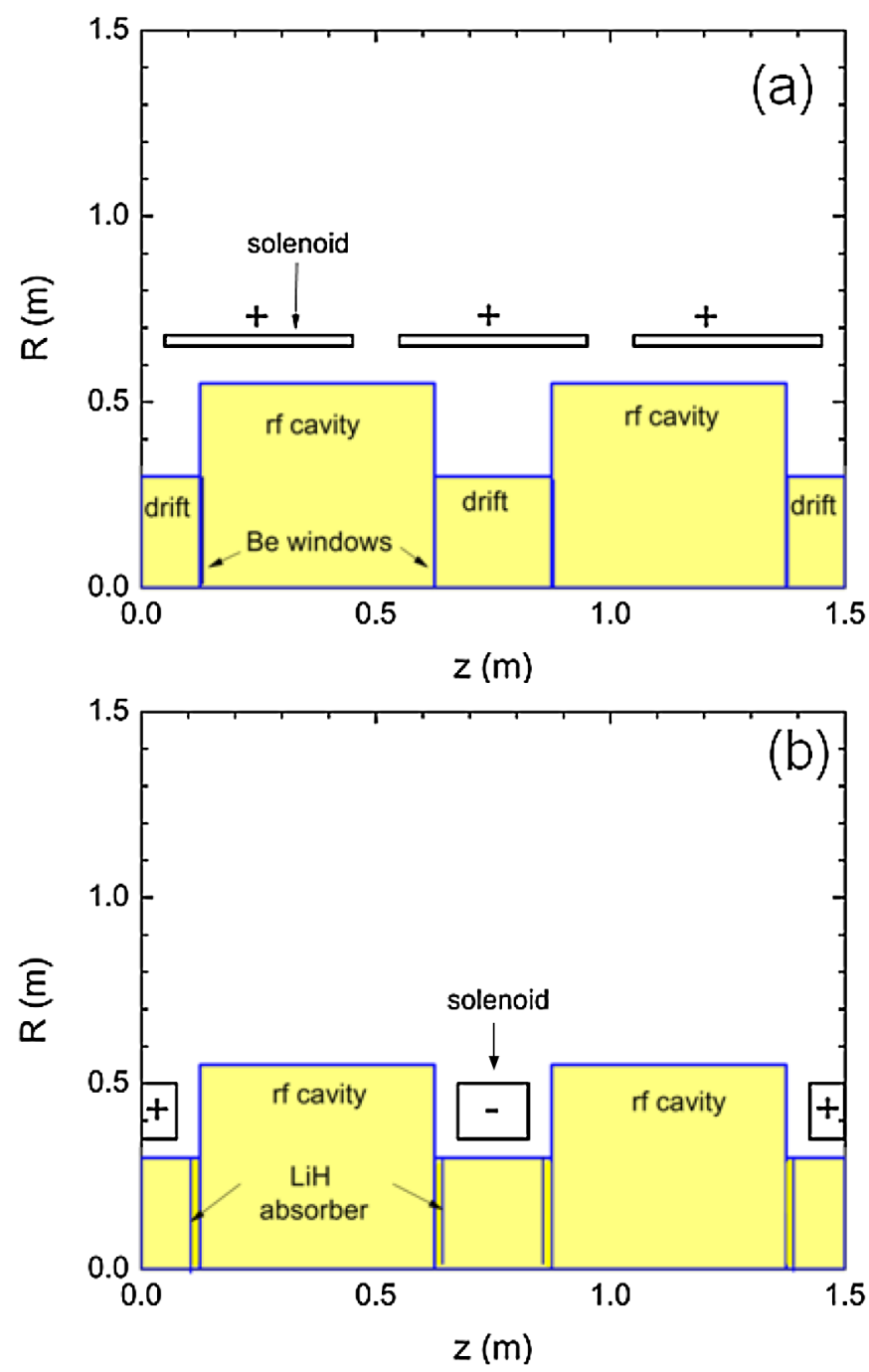

FIG. 2. Two cells of the baseline front-end system for a neutrino factory with conventional pillbox cavities: (a) configuration for the buncher and rotator sections; (b) configuration for the cooling section.
232.0 MHz. In the bunching system, 44 normal-conducting rf cavities are employed, each having a different frequency and the $\mathrm{rf}$ gradient $E_{B}$ increases linearly by

$$
E_{B}(z)=E_{B, \max }\left(\frac{z}{L_{B}}\right)
$$

where $L_{B}$ is the buncher length. In the above expression, $E_{B, \max }$ is the gradient at the end of the buncher and equals $9 \mathrm{MV} / \mathrm{m}$.

In the baseline configuration, the pillbox shaped rf cavities have nominal lengths of $0.5 \mathrm{~m}$ and are separated by a $0.25 \mathrm{~m}$ spacing. To keep the muon beam focused, a constant $1.5 \mathrm{~T}$ solenoidal field is maintained through the buncher. The irises of the pillbox cavities are closed with $200 \mu \mathrm{m}$ thick beryllium (Be) windows. The Be windows roughly double the cavity shunt impedance, and give a higher field on axis for a given amount of rf power [25].

Once the beam leaves the buncher, it consists of a train of bunches with different energies. The beam then is phase rotated with a second string of 56 cavities with decreasing frequencies, but with constant accelerating gradient. The frequencies are chosen so that the centers of the lowenergy bunches increase in energy, while those of the high-energy bunches decrease. The algorithm [24] used for setting this condition is to keep the first reference particle at fixed momentum while uniformly accelerating the second reference particle through the rotator section, so that it attains the first particle's energy at the end of the channel. This is accomplished by increasing slightly the phase shift between the reference particles by $N_{R}=N_{B}+$ 0.05 . With this condition, the bunches are aligned into nearly equal energies over the $42 \mathrm{~m}$ length of the rotator. The rf gradient is kept fixed at $12 \mathrm{MV} / \mathrm{m}$ while the rf frequency drops from 232 to $202 \mathrm{MHz}$. Similar to the buncher, the cavities occupy $0.5 \mathrm{~m}$ over a $0.75 \mathrm{~m}$ long cell and a $1.5 \mathrm{~T}$ solenoidal field continues throughout the section.

Upon exiting the rotator, the muons enter a $\sim 80-100 \mathrm{~m}$ long cooling channel consisting of rf cavities, $\mathrm{LiH}$ absorbers for ionization cooling, and alternating $2.8 \mathrm{~T}$ solenoids for focusing. Figure 2(b) depicts the cooling channel configuration. The cooling section has $0.75 \mathrm{~m}$ long cells and is identical to the cooling scenario used for the International Scoping Study [5]. Each cell has a $0.5 \mathrm{~m}$ long, 201.25 MHz rf pillbox cavity with a gradient of $15 \mathrm{MV} / \mathrm{m}$. The $1.1 \mathrm{~cm}$ thick lithium hydride $(\mathrm{LiH})$ absorbers are part of the cavity windows. $\mathrm{LiH}$ was chosen as the absorber material since it is a low- $Z$ material, and hence has low multiple scattering per unit energy loss and gives good cooling performance. The side of the absorber facing the rf cavity is covered by a thin $300 \mu \mathrm{m}$ Be layer.

The performance of the front-end channel is evaluated by counting the number of simulated particles that fall within a reference acceptance, which approximates the expected acceptance of the downstream accelerator. 
Based on tracking simulations with the ICOOL code [26], at the end of the cooling channel we expect that the system accepts $\sim 0.083$ muons per $8 \mathrm{GeV}$ incident proton, while the transverse rms normalized emittance falls by a factor $\sim 3$ to $\varepsilon_{t} \approx 6.3 \mathrm{~mm}$. The accepted rms longitudinal emittance is $\varepsilon_{l} \approx 61.1 \mathrm{~mm}$.

\section{THE CONCEPT OF MAGNETIC INSULATION}

In the baseline configuration, the rf cavities are closedcell pillbox cavities that operate at relatively high gradients within a few Tesla focusing fields. For example, in the buncher and rotator sections, 200 to $320 \mathrm{MHz}$ rf cavities need to operate in a constant $1.5 \mathrm{~T}$ field with gradients up to $12 \mathrm{MV} / \mathrm{m}$. Two experiments at the MuCool Test Area (MTA) at Fermi National Laboratory explored the efficiency of pillbox rf cavities within magnetic fields: The first had a single pillbox $805 \mathrm{MHz} \mathrm{Cu}$ cavity [15], and the second a single $201 \mathrm{MHz} \mathrm{Cu}$ cavity. In all cases the cavities were "conditioned" prior to achieving the quoted gradients. Conditioning started by slowly ramping up the rf input power. With a constant power level the cavity was allowed to outgas. During the conditioning multipactoring, sparking, dark current, and vacuum fluctuations were monitored. The breakdown gradient was loosely defined to be the limiting gradient above which sparking cannot be eliminated with further conditioning. The sparking rate was $\approx 1 / 3000$ pulses (at $10 \mathrm{~Hz}$ ) at the breakdown gradient [18]. The $805 \mathrm{MHz}$ cavity achieved only $20 \mathrm{MV} / \mathrm{m}$ in a $2 \mathrm{~T}$ field, and $15 \mathrm{MV} / \mathrm{m}$ in a $4 \mathrm{~T}$, even though it ran with a peak gradient at $40 \mathrm{MV} / \mathrm{m}$ in the absence of fields. The $201 \mathrm{MHz}$ cavity without a field achieved $21 \mathrm{MV} / \mathrm{m}$, but in the $0.6 \mathrm{~T}$ fringe field of a $4.5 \mathrm{~T}$ magnet, it only reached $10 \mathrm{MV} / \mathrm{m}$. When the $201 \mathrm{MHz}$ cavity was retested without a field, the cavity could not attain more than $18 \mathrm{MV} / \mathrm{m}$. Therefore, in all cases, operating the rf in magnetic fields caused a decline in the achievable gradient. According to a recent theory [18], this drop in gradient likely occurs after the electrons from a field-emission site are focused by the magnetic field, and damage a surface with high electric fields. Such damage would be caused by fatigue [27] from cyclical strains induced by local heating from the electrons. This theory agrees reasonably well with numerical simulations [19] and with experimental data [15].

Assuming that emitted electrons that are accelerated and focused on other surfaces trigger the breakdown in magnetic fields, the process could be suppressed if the magnetic fields were parallel to all emitting surfaces. Hence, instead of focusing the electrons, the field would return them with little energy to near their points of origin. This idea was recently experimentally tested with a box $805 \mathrm{MHz}$ rf cavity [16,17]. Compared to the case where there was a component of a $0.2 \mathrm{~T}$ field parallel to the rf electric field, the first preliminary results showed a $30 \%$ larger maximum gradient threshold when the magnetic field was introduced at right angles to the rf electric field.
The sparking rate at the maximum gradient was $1 / 20000$ pulses (at $15 \mathrm{~Hz}$ ). Since the IDS-NF baseline employs magnetic fields that have parallel components to the rf electric field that in many cases exceed $0.2 \mathrm{~T}$, there is an argument for considering a lattice with magnetically insulated cavities as an alternative option.

In order to design a magnetically insulated (MI) lattice, we propose placing the primary focus coils in the irises of open multicell cavities, and shaping the walls of the cavity to follow the magnetic-field lines. Figure 3(a) shows a simple example of this principle applied to a single rf cavity with just two coils, one on either side of the cavity. Field emission in such a cavity has been examined numerically and theoretically [21]. Computations showed that electrons emitted from the cavity surface are initially accelerated by the electric field away from that surface. Then, as they attained significant momentum, the magnetic field deflected them and directed them back to the surface.
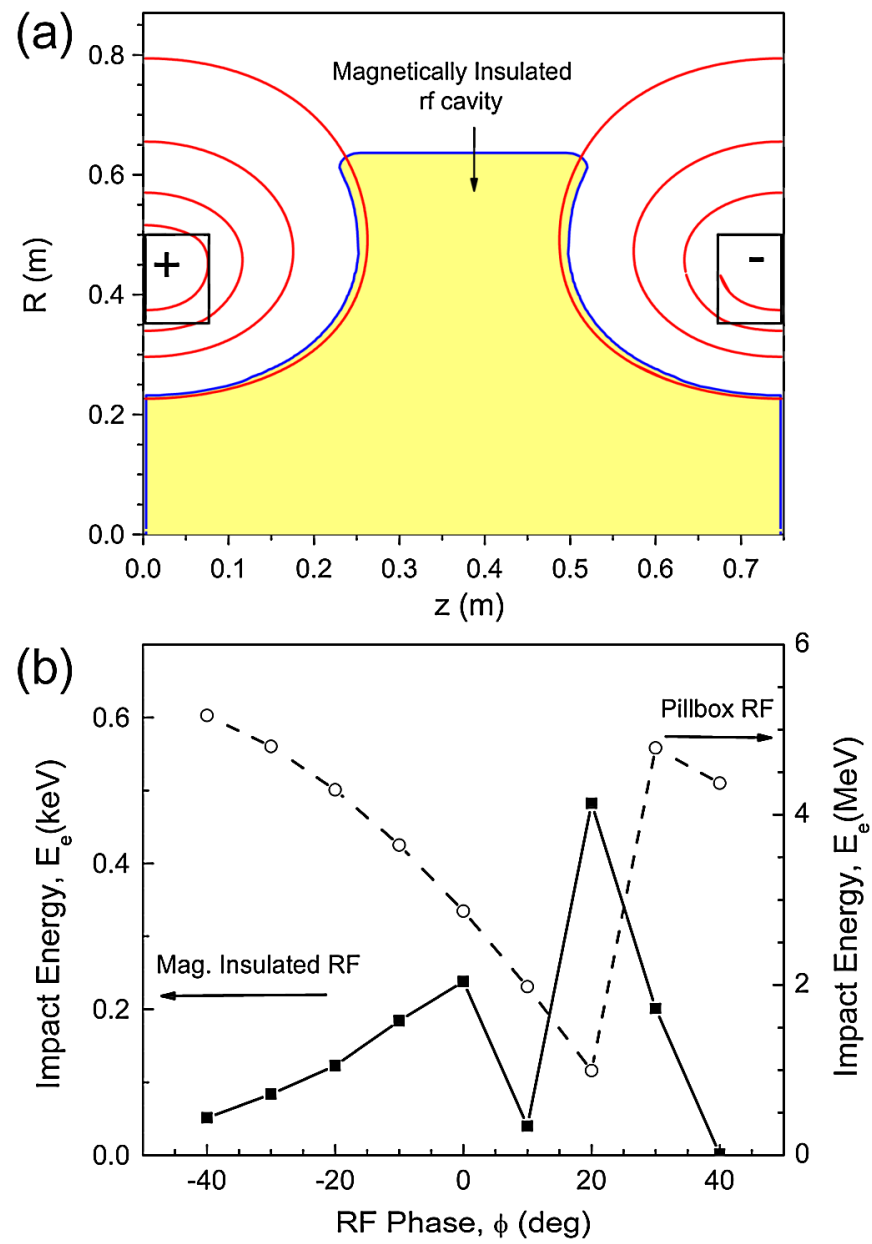

FIG. 3. The principle of rf magnetic insulation: (a) schematic layout of a magnetically insulated $201 \mathrm{MHz}$ cavity for a neutrino factory; (b) the energies of the returning electrons as a function of the rf phase when they are emitted. The solid line shows the energies for a magnetically insulated cavity, while the dashed line shows the energies for a conventional pillbox cavity. 
Compared to conventional pillbox cavities, the simulations indicated that, if a 0.3 T insulation field is applied, the fieldemitted electrons impact the cavity surface with energies that are 4 orders of magnitude lower [Fig. 3(b)]. Compared to $10-40 \mathrm{keV}$, which is the estimated [21] threshold impact energy range for cavity damage assuming $10^{6} \mathrm{rf}$ pulses, the impact data for the insulated case are at least 1 order of magnitude smaller. Thus, with magnetic insulation we expect much less surface damage. Finally, the insulation condition seems insensitive to $1-2 \mathrm{~mm}$ coil misalignments in both horizontal and vertical directions since the simulated electron impact energy for these cases remains below the threshold value.

One possible difficulty that might arise is multipactoring. With magnetic insulation the energy of the impacted electrons for all rf phases is below $0.8 \mathrm{keV}$, which corresponds to the peak secondary emission yield for $\mathrm{Cu}$ [28]. However, small changes in the cavity alignment or in the magnetic-field configuration could lead to increased impact energy and to a resonant condition for the emitted electrons. Thus, the multipactor is a very complex phenomenon and should be investigated in more detail in future studies.

\section{FRONT-END LATTICE WITH MAGNETICALLY INSULATED CAVITIES}

In this section, we explore the option of using a MI lattice for a neutrino factory. In our proposed configuration, both the target and drift sections remain identical to the IDS-NF baseline. In the buncher and rotator, we replace the pillbox cavities by $0.5 \mathrm{~m}$ long magnetically insulated cavities. To align the magnetic-field lines with the cavity surfaces, the focusing coils are brought closer to the beam axis, i.e., the inner radius of the coils is $0.35 \mathrm{~m}$. Because of constraints on the cavity geometry, the coils are spaced $0.60 \mathrm{~m}$ apart, and as a result, there are higher longitudinal harmonics in the axial field. We selected the coil's current density as $35.0 \mathrm{~A} / \mathrm{mm}^{2}$ so that the average field on axis is $1.5 \mathrm{~T}$ (as in the baseline), providing transverse focusing with $\beta_{\perp} \approx 1.17 \mathrm{~m}$. Similar to the baseline configuration, the $\mathrm{rf}$ frequencies decrease from 320 to $232 \mathrm{MHz}$. To assure a more realistic implementation, we grouped several rf cavities together, which leads to fewer different frequencies. A recent study [24] indicated that reducing the number of independent frequencies by a factor of 3 in both the buncher and the rotator does not reduce the accepted muons by more than $\sim 5 \%$. Accordingly, we combine the cavities into triplets with the same frequencies, giving 15 frequencies in the buncher and 18 in the rotator. The cavity centers are equally spaced by $0.75 \mathrm{~m}$ and the rf gradient is set according to Eq. (1). Figure 4(a) shows three cells of our proposed buncher channel, and the corresponding axial magnetic field is depicted in Fig. 4(b). The cavity's iris is covered by a $200 \mu \mathrm{m}$ thick Be window. Although the window is not
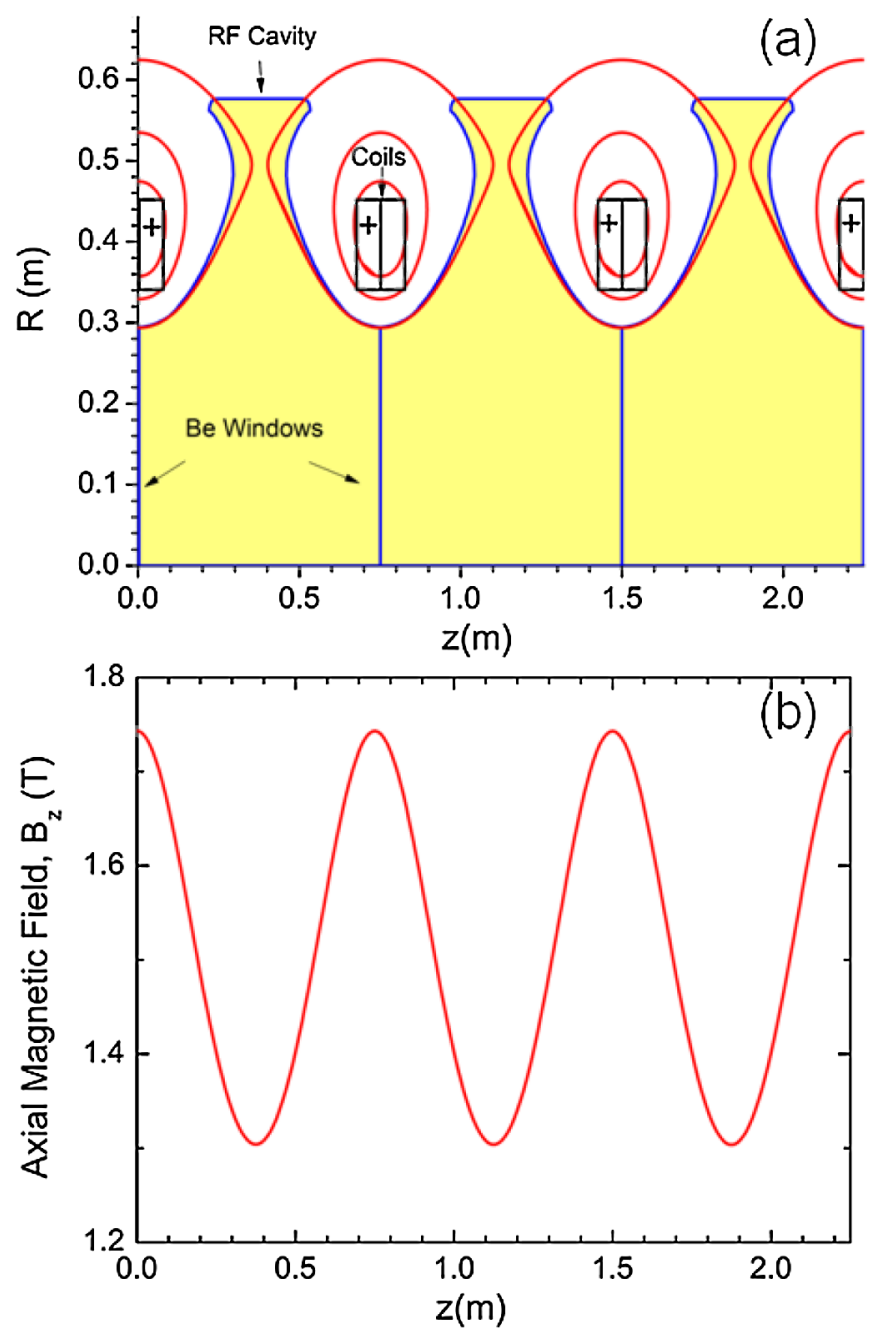

FIG. 4. (a) Three cells of our proposed buncher and rotator sections with magnetically insulated cavities; and, (b) axial magnetic-field profile along the cells.

magnetically insulated, the absence of any damage on Be surfaces in a pillbox cavity [29] suggests that Be is a more resistant material than $\mathrm{Cu}$ to the impact of focused darkcurrent beams. According to a recent theory [18], this is due to its lower density, resulting in less energy deposition per unit volume. Quantitatively, it is expected that for the same rf gradient Be can sustain at least 3.5 times higher magnetic fields than $\mathrm{Cu}[30]$ and thus we consider it likely that the hybrid insulated structure shown in Fig. 4(a) is safe.

The phase-rotator cell is very similar to the buncher cell, with the only difference being that the thickness of the Be windows is $400 \mu \mathrm{m}$. There are 56 cavities in the rotator region with 18 different frequencies that decrease from 232 to $202 \mathrm{MHz}$. All cavities have a fixed gradient equal to $12 \mathrm{MV} / \mathrm{m}$.

Three cells of the proposed MI cooling channel are shown in Fig. 5(a). The total length of the channel is $115 \mathrm{~m}$. As in the baseline design, the lattice consists of a 
sequence of identical $0.75 \mathrm{~m}$ long cells, with $201.25 \mathrm{MHz}$ rf cavities operating at $15 \mathrm{MV} / \mathrm{m}$. One noteworthy difference is that we removed a cavity every third cell, and we extended the ends of the two adjacent cavities. A $2.7 \mathrm{~cm}$ $\mathrm{LiH}$ absorber with a $25 \mu \mathrm{m}$ thick Be coating on both sides is located at the center of the empty cell. Both the current density and position of the coil are like the baseline and generate a $2.8 \mathrm{~T}$ peak field that varies sinusoidally within the channel and provides a minimum transverse beta function $\beta_{\perp} \approx 0.77 \mathrm{~m}$.

In comparison to the baseline design, there are two improvements of our proposed MI cooling channel. First, the absorber is positioned at a location where the transverse beta function is minimum [see Fig. 5(c)]. Since the lattice equilibrium emittance is proportional to the beta function at the absorber [24], at least in theory this configuration should enhance the cooling rate. Second, the Be windows are located within considerably lower rf fields, $E_{w}$ [see Fig. 5(d)] consequently implying less heating at those

(a)
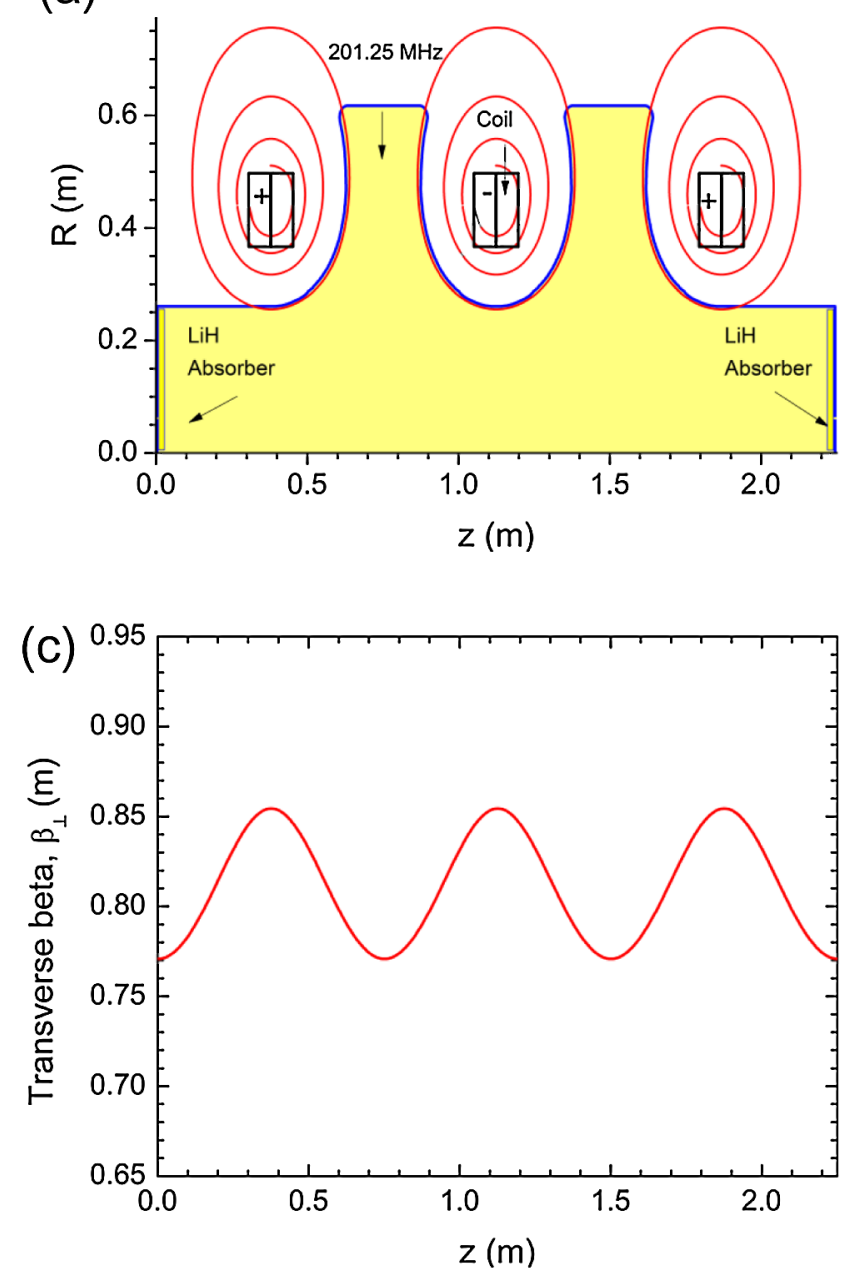

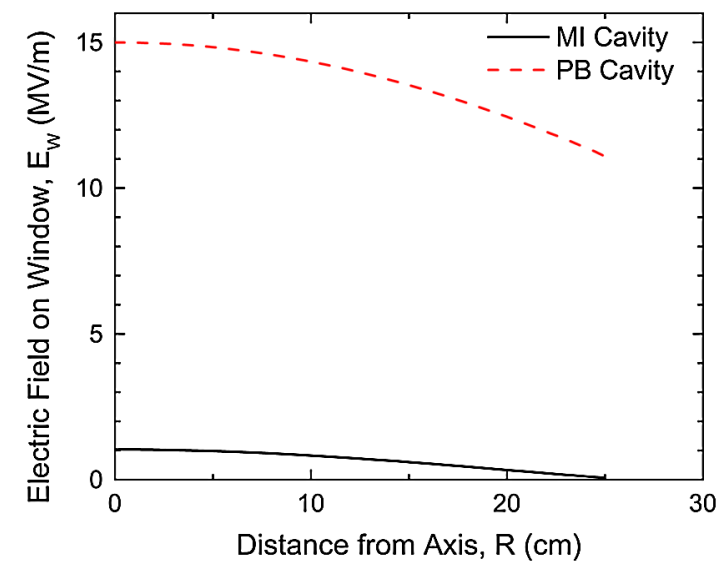

FIG. 6. The rf electric field distributions along the cooler Be window for a channel with magnetically insulated and pillbox cavities. The axis is at $R=0$. Less window heating is anticipated with our proposed magnetically insulated channel since the rf field drops by an order of magnitude.
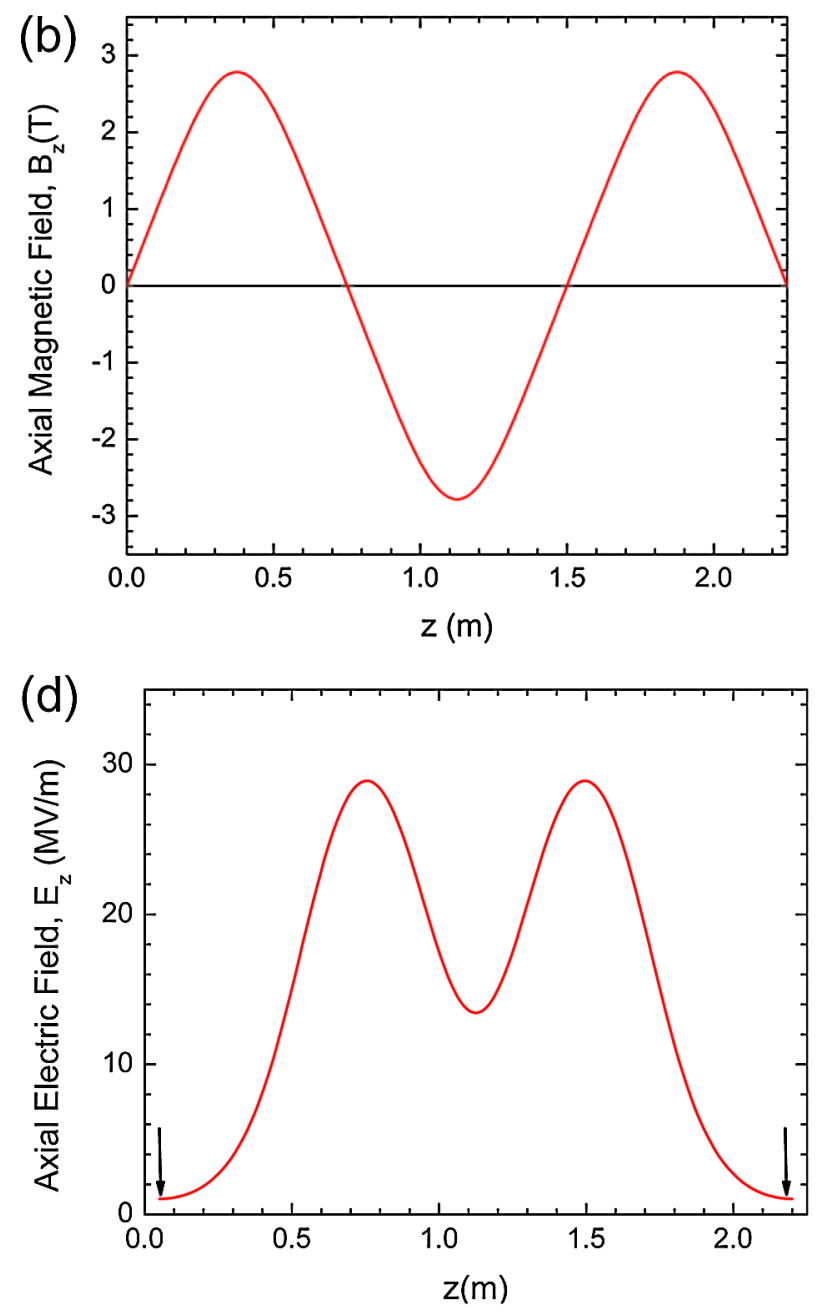

FIG. 5. (a) Three cells of our proposed cooling section with magnetically insulated cavities; (b) axial magnetic-field profile along the cells; (c) transverse beta function along the cells; and (d) on-axis longitudinal rf electric field at various positions along $z$, assuming that the average axial field of each cavity is $15 \mathrm{MV} / \mathrm{m}$. The vertical arrows indicate the locations of the Be windows. 
windows. Note that, if the heating is high enough, melting and differential stresses leading to buckling are possible [6]. Figure 6 compares $E_{w}$ as a function of radius in our proposed lattice with the baseline. The rf field in our case is 1 order of magnitude less. Quantitatively, using a onedimensional solution of the heat conduction equation [31], we predict that the temperature of a $25 \mu \mathrm{m}$ Be layer should be less than $350^{\circ} \mathrm{C}$. This model also predicts that in order to maintain the same temperature in the baseline configuration a $300 \mu \mathrm{m}$ thick window is required. This very thin layer that we propose ensures that the losses on the muon flux due to the windows are less than $1 \%$.

The performance of our front-end channel was simulated using the ICOOL code. Figure 7 shows the evolution of the longitudinal positive muon beam phase space at different locations. Figure 7(a) shows the initial beam distribution at $z=0$, while Fig. 7(b) shows the beam distribution after the drift. Figure 7(c) shows the correlated longitudinal phase space at the exit of the buncher. Note that the beam has been formed into a string of $\sim 200 \mathrm{MHz}$ bunches at different energies. Figure 7(d) exhibits the longitudinal phase space of the beam after the phase-rotator section. The bunches are now aligned into nearly equal energies and the rms energy spread of the beam is reduced to $30 \mathrm{MeV}$, while the average momentum at the exit is $\sim 237 \mathrm{MeV} / \mathrm{c}$. Figure 7(e) shows the longitudinal phase space at the end of the cooling section. The usable beam consists of a $55 \mathrm{~m}$ long train of about 40 bunches with a varying population in each bunch. The mean momentum is $240 \mathrm{MeV} / \mathrm{c}$ with an rms width of $32 \mathrm{MeV} / \mathrm{c}$. Finally, Fig. 7(f) illustrates a few interleaved $\mu^{+}$and $\mu^{-}$bunches exiting the cooling section. Although, the opposite-sign bunches are mostly separated in time, there is a small number of wrong sign particles in the bunch train after
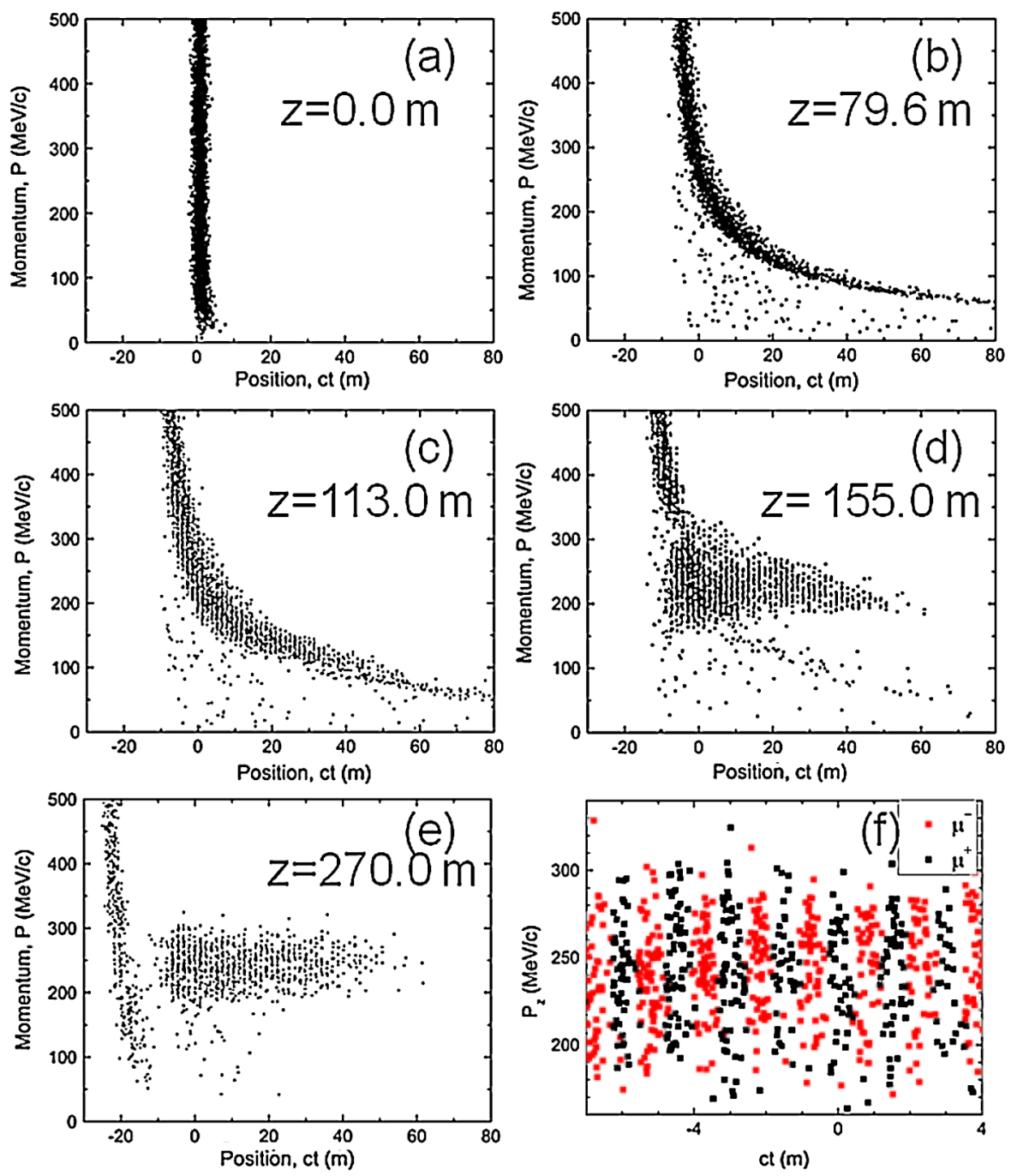

FIG. 7. Simulation results of the evolution of the longitudinal phase space along the drift, buncher, phase rotator, and cooler for our proposed magnetically insulated front-end channel. (a) Pions and muons as produced at the target; (b) muons after the $80 \mathrm{~m}$ drift; (c) at the end of the buncher; (d) at the end of the rotator; (e) at the end of the cooler; (f) a sample from the train of interleaved $\mu^{+}$and $\mu^{-}$ bunches exiting the cooling section. 

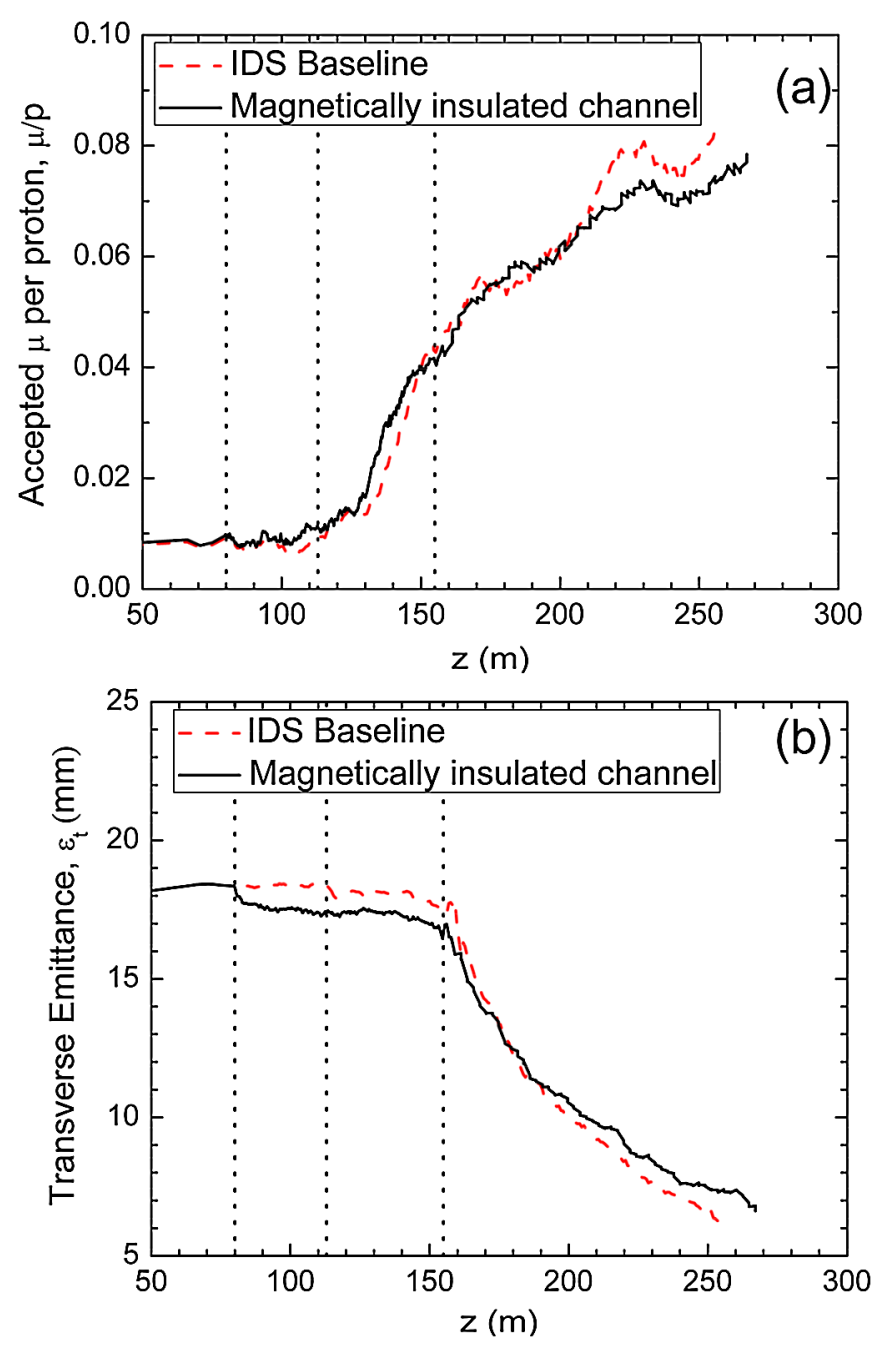

FIG. 8. Simulation results of the performance of a magnetically insulated front-end channel (solid line) vs the baseline (dashed line). (a) The muons per incident proton on the target within a transverse acceptance of $A_{T} \leq 0.03 \mathrm{~m}$ and longitudinal acceptance of $A_{L} \leq 0.15 \mathrm{~m}$, which are the acceptances of the downstream accelerators and storage rings. (b) Normalized transverse rms emittance. cooling, but these will be separated by the dipoles in the subsequent accelerators and storage ring [5].

Simulations of the overall performance of our proposed MI front-end lattice are displayed in Fig. 8 (solid line) and summarized in more detail in Table I. We compare these data against the baseline in Sec. II (dashed line). Figure 8 (a) shows the number of produced muons per incident proton that fit into the transverse and longitudinal acceptance. Figure 8(b) illustrates the cooling in normalized transverse emittance along the channel. The cooling channel produces a final value of $6.7 \mathrm{~mm}$, i.e., almost a factor of 3 less than its initial value. In addition, the channel achieves $\sim 0.078$ muon per proton for each sign of muons. The final values of $\varepsilon_{t}$ and $\mu / p$ meet the IDS-NF requirements.

Figure 9 examines the consequences of using lower rf fields for the cooler section. As the plot indicates, a lower gradient would clearly hurt the front-end performance. For instance, a drop from 15 to $12 \mathrm{MV} / \mathrm{m}$ would reduce the number of accepted muons by $12 \%-15 \%$. On the other hand, for gradients higher than $15 \mathrm{MV} / \mathrm{m}$ the MI lattice appears to have worse performance than a lattice with pillboxes. However, at the baseline gradient of $15 \mathrm{MV} / \mathrm{m}$ the difference is just 5\% and, most likely, the primary reason for this discrepancy is the rapid variation of the axial accelerating rf gradient within a MI cooling lattice [see Fig. 5(d)].

The viability of the current IDS-NF baseline for a neutrino factory largely depends on whether the rf pillbox cavities work in a magnetic field. It must be proven experimentally that the $201 \mathrm{MHz}$ rf pillbox cavities at $15 \mathrm{MV} / \mathrm{m}$ do not break down in magnetic fields of up to 2 T. If pillbox cavities cannot be used with the design gradients, a MI cooling channel offers an alternative that shields the cavities from those strong fields. Compared to a conventional lattice with pillboxes, simulations suggested that a MI lattice can be employed without significant losses in performance, especially at the baseline gradient.

TABLE I. Parameters of two front-end scenarios: IDS-NF baseline with pillbox cavities (left) and magnetically insulated channel (right).

\begin{tabular}{lcc}
\hline \hline & IDS-NF baseline & Magnetically insulated channel \\
\hline Drift length $(\mathrm{m})$ & 79.6 & 79.6 \\
Buncher length $(\mathrm{m})$ & 33.0 & 33.0 \\
Buncher rf frequencies & $320 \rightarrow 232 \mathrm{MHz}$ & $320 \rightarrow 232 \mathrm{MHz}$ \\
Buncher rf gradient $(\mathrm{MV} / \mathrm{m})$ & $0-9$ & $0-9$ \\
Rotator length $(\mathrm{m})$ & 42.0 & 42.0 \\
Rotator rf frequencies & $232 \rightarrow 202 \mathrm{MHz}$ & $232 \rightarrow 202 \mathrm{MHz}$ \\
Rotator rf gradient $(\mathrm{MV} / \mathrm{m})$ & 12 & 12 \\
Cooler length $(\mathrm{m})$ & 100 & 115 \\
Cooler rf gradient $(\mathrm{MV} / \mathrm{m})$ & 15.0 & 15.0 \\
Accepted $\mu / \mathrm{p}$ for $8 \mathrm{GeV}$ p & 0.083 & 0.078 \\
Final transverse emittance $(\mathrm{mm})$ & 6.3 & 6.3 \\
rf Be windows buncherlrotator & $200 \mu \mathrm{m} \backslash 400 \mu \mathrm{m}$ & $200 \mu \mathrm{m} \backslash 400 \mu \mathrm{m}$ \\
\hline \hline
\end{tabular}


TABLE II. Characteristics of the rf system.

\begin{tabular}{lcc}
\hline \hline & Pillbox & Magnetically insulated \\
\hline rf frequency $[\mathrm{MHz}]$ & 201 & 201 \\
Cavity radius $[\mathrm{cm}]$ & 60.7 & 64.0 \\
Shunt impedance $[\mathrm{M} \Omega / \mathrm{m}]$ & 30.3 & 15.5 \\
$B_{\max } / E_{\max }[\mathrm{mT} /(\mathrm{MV} / \mathrm{m})]$ & 2.1 & 2.9 \\
$E_{\max } / E_{0}$ & 1.04 & 1.04 \\
\hline \hline
\end{tabular}

Power demand may be a drawback of a lattice with magnetically insulated rf cavities. The IDS-NF rf cavities on the cooler are $\mathrm{Cu}$ pillboxes (at $201 \mathrm{MHz}, Q=53000$, and radius $=0.60 \mathrm{~m}$ ) and are described in Table II. According to the IDS-NF power requirement estimates [24], the buncher needs cavities with powers ranging from 0.5-3.5 MW. The rotator requires 56 cavities with 2.5 MW power per cavity, while the cooling channel needs about 100 cavities with 4 MW power per cavity. Earlier, we examined [21] the rf properties of magnetically insulated cavities. Compared to pillboxes, we demonstrated that MI cavities had half the shunt input impedance (see Table II). Since the power scales as one over the shunt impendence, it is evident that MI cavities require at least twice the amount of power. We summarize the approximate rf requirements for a MI channel in Table III.

\section{SUMMARY}

It has been suggested computationally and experimentally that the maximum achievable gradient is enhanced by introducing an external magnetic field at right angles to the rf electric field since this suppresses field-emission processes. Here, we have discussed a possible scheme for extending the concept of magnetic insulation to capture, transport, and cool muons in a neutrino factory. We incorporated this idea into a new lattice design where the rf cavities are shaped so that their walls were tangential to the magnetic-field lines. We showed that, with magnetic insulation, the field-emitted electrons impact the cavity surface with energies 4 orders of magnitude less than in conventional pillbox cavities; consequently, damage from field emission is suppressed significantly. The goal of this work was to present the concept of magnetic insulation for a particle accelerator application, explain its basic features, and verify the scheme by numerical simulations. While demanding in terms of power requirements, this neutrino factory lattice showed satisfactory performance in both

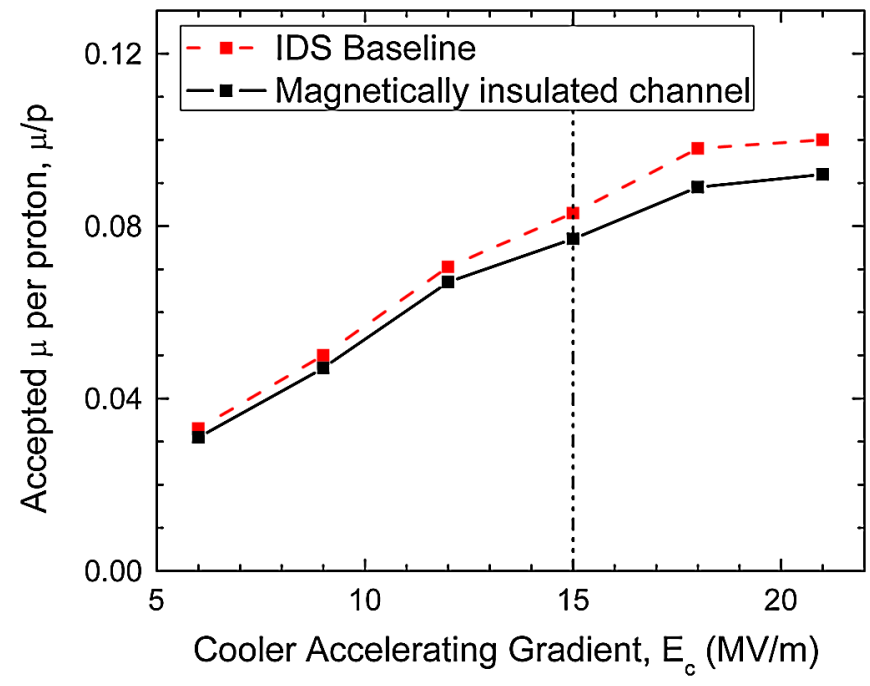

FIG. 9. Simulation results of the effect of maximum rf gradient on the neutrino factory front end. Note that both buncher and rotator gradients remain fixed as described in Table I. The dashed vertical line shows the baseline accelerating gradient.

cooling and collecting the accepted muons within the requirements for the IDS-NF. Optimizations were also made to reduce heating on the absorber windows, minimizing losses in the accepted muon fluxes.

It is critically important to the development of a MI front-end lattice for a Neutrino Factory that a well thought-out program be pursued. This program should begin with simple experiments to verify the concept. In collaboration with Fermilab, one experiment [17] is already in progress and another one is under consideration. For the first step a box-shaped $805 \mathrm{MHz}$ cavity has been designed and built so that it can be mounted in the center of an up to $4 \mathrm{~T}$ superconducting magnet and tested with the magnetic fields perpendicular to the rf electric field. The next step will be to tilt the cavity with respect to the magnetic field and study the limit of stable operating gradient as a function of angle. In order to verify reproducibility, a second identical cavity is currently under construction. Although a rectangular cavity placed in a uniform field provides the simplest demonstration of the principle, a test of a cavity for a muon cooling lattice would need to place the primary focus coils in the irises of an open multicell structure and shape the walls of the cavity to follow the magnetic-field lines [see Fig. 3(a)]. Such a cavity would form the basis of a demonstration experiment specifically for a muon accelerator application, and a

TABLE III. Approximate rf requirements of a magnetically insulated front-end channel.

\begin{tabular}{lccc}
\hline \hline Region & Number of rf cavities & rf frequencies & rf gradient/peak power per cavity \\
\hline Buncher & 44 & 320 to $232 \mathrm{MHz}$ & $0-9 \mathrm{MV} / \mathrm{m} / \geq 1-7 \mathrm{MW}$ \\
Rotator & 56 & 232 to $202 \mathrm{MHz}$ & $12 \mathrm{MV} / \mathrm{m} / \geq 5 \mathrm{MW}$ \\
Cooler & 104 & $201 \mathrm{MHz}$ & $15 \mathrm{MV} / \mathrm{m} / \geq 8 \mathrm{MW}$ \\
\hline \hline
\end{tabular}


preliminary experimental configuration can be found in Refs. $[18,21]$. A successful demonstration will provide us with a new versatile tool for a future muon accelerator.

\section{ACKNOWLEDGMENTS}

The authors are grateful to J. T. Keane, J. Norem, J. S. Berg, and H. Kirk for useful discussions. This work is supported by the U.S. Department of Energy, Contract No. DE-AC02-98CH10886.

[1] Y. Fukuda et al., Phys. Rev. Lett. 81, 1562 (1998).

[2] S. Geer, Phys. Rev. D 57, 6989 (1998).

[3] D. Cline and D. Neuffer, AIP Conf. Proc. 68, 856 (1981).

[4] A. Blondel et al., Report No. CERN 2004-002 ECFA/ CERN, 2004.

[5] M. Apollonio et al., JINST 4, P07001 (2009).

[6] J. S. Berg et al., Phys. Rev. ST Accel. Beams 9, 011001 (2006).

[7] C. Ankenbrandt, S.A. Bogacz, A. Bross, S. Geer, C. Johnstone, D. Neuffer, and M. Popovic, Phys. Rev. ST Accel. Beams12, 070101 (2009).

[8] S. Ozaki, R. Palmer, M. Zisman, and J. Gallardo, BNL52623 (2001).

[9] N. Holtkamp and D. Finley, Report No. Fermilab-Pub-00/ 108-e, 2000.

[10] C. Albright et al., arXiv:physics/0411123v2.

[11] Y. Mori, J. Phys. G 29, 1527 (2003).

[12] A. De Rujula, M. B. Gavela, and P. Hernandez, Nucl. Phys. B547, 21 (1999).

[13] K. T. McDonald et al., in Proceedings of the 23rd Particle Accelerator Conference, Vancouver, Canada, 2009 (IEEE, Piscataway, NJ, 2009), TU4GRRI03.

[14] D. Neuffer, Nucl. Instrum. Methods Phys. Res., Sect. A 532, 26 (2004).

[15] A. Moretti, Z. Qian, J. Norem, Y. Torun, D. Li, and M. Zisman, Phys. Rev. ST Accel. Beams 8, 072001 (2005).
[16] For example, slide 4 in A. Moretti, at Presentation at the MAP Weekly Meeting, Fermilab, 2010, http://indico.fnal .gov/conferenceDisplay.py?confId=3396.

[17] Y. Torun et al., in Proceedings of the 1st International Particle Accelerator Conference, Kyoto, Japan (2010), THPEA054.

[18] R. B. Palmer, R. C. Fernow, J. C. Gallardo, D. Stratakis, and D. Li, Phys. Rev. ST Accel. Beams 12, 031002 (2009).

[19] D. Stratakis, J.C. Gallardo, and R. B. Palmer, Nucl. Instrum. Methods Phys. Res., Sect. A 620, 147 (2010).

[20] F. Winterberg, Nature (London) 246, 299 (1973).

[21] D. Stratakis, J. C. Gallardo, and R. B. Palmer, J. Phys. G 37, 105011 (2010).

[22] C. Rogers, Proceedings of the 11th International Workshop on Neutrino Factories, Super Beams and Beta Beams, 2009 (AIP Press, Chicago, IL, 2009), Vol. 1222, p. 298.

[23] G. Prior, Proceedings of the 11th International Workshop on Neutrino Factories, Super Beams and Beta Beams, 2009 (AIP Press, Chicago, IL, 2009), Vol. 1222, p. 332.

[24] D. Neuffer, M. Martini, G. Prior, C. T. Rogers, and C. Y. Yoshikawa, Proceedings of the 1st International Particle Accelerator Conference, Kyoto, Japan (2010), WEPE068.

[25] D. Li et al., J. Phys. G 29, 1683 (2003).

[26] R.C. Fernow, Proceedings of the 1999 Particle Accelerator Conference, New York (IEEE, New York, 1999), p. 3020.

[27] D. P. Pritzkau and R. H. Siemann, Phys. Rev. ST Accel. Beams 5, 112002 (2002).

[28] L. Ge et al., Proceedings of the 23rd Particle Accelerator Conference, Vancouver, Canada, 2009 (Ref. [13]), WE5PFP020.

[29] D. Li et al., in Proceedings of the 20th Particle Accelerator Conference, Portland, OR, 2003 (IEEE, New York, 2003), p. 1246.

[30] For example, see Fig. 6(b) in D. Stratakis, J. C. Gallardo, and R. B. Palmer, Proceedings of the 11th International Workshop on Neutrino Factories, Super Beams and Beta Beams, 2009 (AIP Press, Chicago, IL, 2009), Vol. 1222, p. 303.

[31] R. C. Fernow, Report No. MUC-NOTE-COOL_THEORY317, 2005. 